УДК 515.537.6

O. M. Mulyava

\title{
ON BELONGING OF ENTIRE DIRICHLET SERIES TO A MODIFIED GENERALIZED CONVERGENCE CLASS
}

O. M. Mulyava. On belonging of entire Dirichlet series to a modified generalized convergence class, Mat. Stud. 50 (2018), 135-142.

For entire Dirichlet series $F(s)=\sum_{n=0}^{+\infty} a_{n} e^{s \lambda_{n}}$ we found conditions on $a_{n}, \lambda_{n}$ and on positive functions $\alpha$ and $\beta$ continuous increasing to $+\infty$ on $[0,+\infty)$ are found, under which the condition $\int_{\sigma_{0}}^{+\infty} \frac{1}{\beta(\sigma)} \alpha\left(\frac{1}{\sigma} \ln M(\sigma, F)\right) d \sigma<+\infty$ is equivalent to the condition

$$
\sum_{n=1}^{+\infty}\left(\alpha\left(\lambda_{n}\right)-\alpha\left(\lambda_{n-1}\right)\right) \beta_{1}\left(\frac{1}{\lambda_{n}} \ln \frac{1}{\left|a_{n}\right|}\right)<+\infty
$$

where $\beta_{1}(x)=\int_{x}^{+\infty} \frac{d t}{\beta(t)}$, and $M(\sigma, F)=\sup \{|F(\sigma+i t)|: t \in \mathbb{R}\}$.

1. Introduction. G. Valiron [1, p.18] proved that if an entire function $f(z)=\sum_{n=0}^{+\infty} a_{n} z^{n}$ has the order $\varrho \in(0,+\infty)$ and belongs to the convergence class, that is

$$
\int_{1}^{+\infty} \frac{\ln M_{f}(r)}{r^{\varrho+1}}<+\infty, \quad M_{f}(r):=\max \{|f(z)|:|z|=r\},
$$

then

$$
\sum_{n=1}^{+\infty}\left|a_{n}\right|^{\varrho / n}<+\infty
$$

This result was generalized in $[2,3]$ for the case of entire (absolutely convergent in $\mathbb{C}$ ) Dirichlet series of the form

$$
F(s)=a_{0}+\sum_{n=1}^{+\infty} a_{n} e^{s \lambda_{n}}, \quad 0<\lambda_{n} \uparrow+\infty(1 \leq n \uparrow+\infty) .
$$

It was proved ( [3, Theorem 2]) that if entire Dirichlet series (1) has $R$-order $\varrho_{R}=\varrho \in$ $(0,+\infty)$ and $\ln n=O\left(\lambda_{n}\right)$ as $n \rightarrow+\infty$, then in order that relation

$$
\int_{0}^{+\infty} \frac{\ln M(\sigma, F)}{r^{\varrho+1}}<+\infty, \quad M(\sigma, F):=\sup \{|F(\sigma+i t)|: t \in \mathbb{R}\},
$$

2010 Mathematics Subject Classification: 30B50.

Keywords: entire Dirichlet series; convergence class.

doi:10.15330/ms.50.2.135-142

(C) O. M. Mulyava, 2018 
be satisfied it is necessary and in the case when $\varkappa_{n}(F):=\frac{\ln \left|a_{n}\right|-\ln \left|a_{n+1}\right|}{\lambda_{n+1}-\lambda_{n}} \nearrow+\infty$ as $n \rightarrow+\infty$ it is sufficient that

$$
\sum_{n=1}^{+\infty}\left(\lambda_{n}-\lambda_{n-1}\right)\left|a_{n}\right|^{\varrho / \lambda_{n}}<+\infty .
$$

In [2], this result was proved by additional assumption that the sequence $\left(\lambda_{n}\right)$ has a positive finite step, that is $0<h \leq \lambda_{n+1}-\lambda_{n} \leq H<+\infty$ for $n \geq 0$.

In papers [3,4], we also proved the results about belonging of Dirichlet series to so-called the generalized convergence $\alpha \beta$-class. To give its definition we denote, as in [5], by $L$ the class of continuous nonnegative on $(-\infty,+\infty)$ functions $\alpha$ such that $\alpha(x)=\alpha\left(x_{0}\right) \geq 0$ for $x \leq x_{0}$ and $\alpha(x) \uparrow+\infty$ as $x \rightarrow+\infty$. We say that $\alpha \in L^{0}$, if $\alpha \in L$ and $\alpha((1+o(1)) x)=(1+o(1)) \alpha(x)$ as $x \rightarrow+\infty$.

The following theorem is proved in [4].

Theorem 1 ([4]). Let $\alpha$ be a concave function on $\left[x_{0},+\infty\right)$ and $\alpha\left(e^{x}\right) \in L^{0}$, and a function $\beta \in L^{0}$ satisfies the conditions $x \beta^{\prime}(x) / \beta(x) \geq h>0$ for $x \geq x_{0}$ and

$$
\int_{x_{0}}^{+\infty} \frac{\alpha(x)}{\beta(x)} d x<+\infty
$$

Suppose that the exponents $\lambda_{n}$ of entire Dirichlet series (1) satisfies the condition $\ln n=$ $o\left(\lambda_{n} \beta^{-1}\left(\alpha\left(\lambda_{n}\right)\right)\right)$ as $n \rightarrow+\infty$. In order that

$$
\int_{\sigma_{0}}^{+\infty} \frac{\alpha(\ln M(\sigma, F))}{\beta(\sigma)} d \sigma<+\infty
$$

it is necessary and in the case, when $\varkappa_{n}(F) \nearrow+\infty, n \rightarrow+\infty$, it is sufficient that

$$
\sum_{n=1}^{+\infty}\left(\alpha\left(\lambda_{n}\right)-\alpha\left(\lambda_{n-1}\right)\right) \beta_{1}\left(\frac{1}{\lambda_{n}} \ln \frac{1}{\left|a_{n}\right|}\right)<+\infty, \quad \beta_{1}(x)=\int_{x}^{+\infty} \frac{d t}{\beta(t)} .
$$

Besides the generalized convergence $\alpha \beta$-class defined by condition (4) for entire Dirichlet series we also define the modified generalized convergence $\alpha \beta$-class by the condition

$$
\left(\exists \sigma_{0}>0\right): \quad \int_{\sigma_{0}}^{+\infty} \frac{1}{\beta(\sigma)} \alpha\left(\frac{\ln M(\sigma, F)}{\sigma}\right) d \sigma<+\infty
$$

for $\alpha \in L$ and $\beta \in L$. Here we obtain an analog of Theorem 1 for the modified generalized convergence $\alpha \beta$-class.

2. Modified generalized convergence $\alpha \beta$-class in terms of maximal term. For entire Dirichlet series (1) let $\mu(\sigma, F)=\max \left\{\left|a_{n}\right| \exp \left\{\sigma \lambda_{n}\right\}: n \geq 0\right\}$ be its maximal term and $\nu(\sigma)=\max \left\{n:\left|a_{n}\right| \exp \left\{\sigma \lambda_{n}\right\}=\mu(\sigma, F)\right\}$ be its central index.

At first, we investigate conditions under which restriction (5) is equivalent to the condition

$$
\left(\exists \sigma_{0}>0\right): \quad \int_{\sigma_{0}}^{+\infty} \frac{1}{\beta(\sigma)} \alpha\left(\frac{\ln \mu(\sigma, F)}{\sigma}\right) d \sigma<+\infty .
$$

We need the following properties of the functions from $L^{0}$ proved in [6, p. 25] and [7]. 
Lemma $1([6,7])$. Let $\alpha \in L$ and $B(\varepsilon)=\varlimsup_{x \rightarrow+\infty} \frac{\alpha((1+\varepsilon) x)}{\alpha(x)}, \varepsilon>0$. In order that $\alpha \in L^{0}$, it is necessary and sufficient that $B(\varepsilon) \rightarrow 1$ as $\varepsilon \rightarrow 0$.

If $\alpha \in L^{0}$ then $\alpha$ is $R O$-increasing ([8]), i.e. for every $h \in[1, a], 1<a<+\infty$, and all $x \geq x_{0}$ the inequality $\frac{\alpha(h x)}{\alpha(x)} \leq M(a)<+\infty$ holds.

Using this lemma we prove the following theorem.

Theorem 2. Let $\alpha \in L^{0}$ and $\beta(x)=x \gamma(x), \gamma \in L^{0}$. If

$$
\ln n=O\left(\lambda_{n} \gamma^{-1}\left(\alpha\left(\lambda_{n}\right)\right)\right), \quad n \rightarrow+\infty,
$$

then conditions (5) and (6) are equivalent.

Proof. In view of Cauchy's inequality $\mu(\sigma, F) \leq M(\sigma, f)$ from (5) we have (6). On the other hand (see $[9, \mathrm{p} .182]$ and $[6, \mathrm{p} .17])$,

$$
\ln \mu(\sigma, F)=\ln \mu\left(\sigma_{0}, F\right)+\int_{\sigma_{0}}^{\sigma} \lambda_{\nu(t, F)} d t .
$$

Since $\lambda_{\nu(\sigma, F)}$ is nondecreasing function, the function $\frac{1}{\sigma} \int_{\sigma_{0}}^{\sigma} \lambda_{\nu(t, F)} d t\left(0 \leq \sigma_{0} \leq \sigma<+\infty\right)$ is also nondecreasing. Therefore, in view of the condition $\alpha \in L^{0}$ from (8) we have

$$
\alpha\left(\frac{\ln \mu(\sigma, F)}{\sigma}\right)=(1+o(1)) \alpha\left(\frac{1}{\sigma} \int_{\sigma_{0}}^{\sigma} \lambda_{\nu(t, F)} d t\right), \quad \sigma \rightarrow+\infty,
$$

and in view of (8) for every $\varepsilon>0$ and for all $\sigma \geq \sigma_{0}(\varepsilon)$

$$
\begin{gathered}
\left.\varepsilon>\int_{\sigma}^{+\infty} \frac{1}{\beta(t}\right) \alpha\left(\frac{\ln \mu(t, F)}{t}\right) d t \geq(1+o(1)) \alpha\left(\frac{1}{\sigma} \int_{\sigma_{0}}^{\sigma} \lambda_{\nu(t, F)} d t\right) \int_{\sigma}^{+\infty} \frac{d x}{x \gamma(x)} \geq \\
\geq(1-\varepsilon) \alpha\left(\frac{\ln \mu(\sigma, F)}{\sigma}\right) \int_{\sigma}^{2 \sigma} \frac{d x}{x \gamma(x)} \geq(1-\varepsilon) \alpha\left(\frac{\ln \mu(\sigma, F)}{\sigma}\right) \frac{\ln 2}{\gamma(2 \sigma)},
\end{gathered}
$$

whence

$$
\alpha\left(\frac{\ln \mu(\sigma, F)}{\sigma}\right) \leq \varepsilon_{1} \gamma(2 \sigma), \quad \varepsilon_{1}=\frac{\varepsilon}{(1-\varepsilon) \ln 2} \rightarrow 0(\varepsilon \rightarrow 0),
$$

for all large enough $\sigma$ and, thus,

$$
\ln \left|a_{n}\right| \leq \ln \mu(\sigma, F)-\sigma \lambda_{n} \leq \sigma\left(\alpha^{-1}\left(\varepsilon_{1} \gamma(2 \sigma)\right)-\lambda_{n}\right)
$$

for all $n \geq 0$ and for all large enough $\sigma$. We choose $\sigma=\sigma_{n}=\frac{1}{2} \gamma^{-1}\left(\frac{1}{\varepsilon_{1}} \alpha\left(\frac{\lambda_{n}}{2}\right)\right)$. Then

$$
\ln \left|a_{n}\right| \leq-\frac{\lambda_{n}}{2} \sigma_{n}=-\frac{\lambda_{n}}{4} \gamma^{-1}\left(\frac{1}{\varepsilon_{1}} \alpha\left(\frac{\lambda_{n}}{2}\right)\right) .
$$

Since $\alpha \in L^{0}$, by Lemma $1 \alpha(\lambda) \leq M \alpha(\lambda / 2)$ and, therefore,

$$
-\ln \left|a_{n}\right| \geq \frac{\lambda_{n}}{4} \gamma^{-1}\left(\frac{1}{M \varepsilon_{1}} \alpha\left(\lambda_{n}\right)\right)
$$


i. e.

$$
\begin{gathered}
\varlimsup_{n \rightarrow+\infty} \frac{\ln n}{-\ln \left|a_{n}\right|} \leq 4 \varlimsup_{n \rightarrow+\infty}\left(\frac{\ln n}{\lambda_{n} \gamma^{-1}\left(\alpha\left(\lambda_{n}\right)\right)} \frac{\lambda_{n} \gamma^{-1}\left(\alpha\left(\lambda_{n}\right)\right)}{\lambda_{n} \gamma^{-1}\left(\frac{1}{M \varepsilon_{1}} \alpha\left(\lambda_{n}\right)\right)}\right) \leq \\
\leq 4 \varlimsup_{n \rightarrow+\infty} \frac{\ln n}{\lambda_{n} \gamma^{-1}\left(\alpha\left(\lambda_{n}\right)\right)} \varlimsup_{x \rightarrow+\infty} \frac{\gamma^{-1}\left(M \varepsilon_{1} x\right)}{\gamma^{-1}(x)} .
\end{gathered}
$$

We put $A(\varepsilon)=\varlimsup_{x \rightarrow+\infty} \frac{\gamma^{-1}(\varepsilon x)}{\gamma^{-1}(x)}$ and prove that $A(\varepsilon) \rightarrow 0$ as $\varepsilon \rightarrow 0$. Indeed, the function $A(\varepsilon)$ does not decrease on $\left(0, \varepsilon_{0}\right]$. Therefore, if $A(\varepsilon) \not \rightarrow 0$ as $\varepsilon \rightarrow 0$ then $A(\varepsilon) \geq h>0$ and there exists an increasing to $+\infty$ sequence $\left(x_{k}\right)$ such that $\frac{\gamma^{-1}\left(\varepsilon x_{k}\right)}{\gamma^{-1}\left(x_{k}\right)} \geq h / 2$, i. e. by Lemma 1 $\varepsilon x_{k} \geq \gamma\left(h \gamma^{-1}\left(x_{k}\right) / 2\right) \geq x_{k} / M$. It is impossible in view of the arbitrariness of $\varepsilon$. Thus, in view of (7) we have $\varlimsup_{n \rightarrow+\infty} \frac{\ln n}{-\ln \left|a_{n}\right|}=0$. In [6, p. 23] it is proved that if $\varlimsup_{n \rightarrow+\infty} \frac{\ln n}{-\ln \left|a_{n}\right|}=h_{0}<1$ then for every $\varepsilon \in\left(0,1-h_{0}\right)$ there exists $A_{0}(\varepsilon) \in(0,+\infty)$ such that for all $\sigma>0$ the inequality

$$
M(\sigma, F) \leq A_{0}(\varepsilon) \mu\left(\frac{\sigma}{1-h_{0}-\varepsilon}, F\right) .
$$

holds. Since now $h_{0}=0$, by this statement one has $\ln M(\sigma, F) \leq \ln \mu((1+\varepsilon) \sigma, F)+A_{1}(\varepsilon)$ for each $\varepsilon \in(0,1)$, all $\sigma>0$ and some $A_{1}(\varepsilon)<+\infty$. Hence, it follows that

$$
\begin{gathered}
\int_{\sigma_{0}}^{+\infty} \frac{1}{\beta(\sigma)} \alpha\left(\frac{\ln M(\sigma, F)}{\sigma}\right) d \sigma \leq \int_{\sigma_{0}}^{+\infty} \frac{1}{\beta(\sigma)} \alpha\left(\frac{\ln \mu((1+\varepsilon) \sigma, F)+A_{1}(\varepsilon)}{\sigma}\right) d \sigma= \\
\quad=\int_{\sigma_{0}}^{+\infty} \frac{1}{\beta((1+\varepsilon) \sigma)} \alpha\left((1+\varepsilon) \frac{\ln \mu((1+\varepsilon) \sigma, F)+A_{1}(\varepsilon)}{(1+\varepsilon) \sigma}\right) \frac{\beta((1+\varepsilon) \sigma)}{\beta(\sigma)} d \sigma .
\end{gathered}
$$

Using Lemma 1, the last inequality and (6), in view of the conditions $\alpha \in L^{0}, \beta(x)=x \gamma(x)$ and $\gamma \in L^{0}$ imply correlation (5).

3. Main theorem. To description the belonging of entire Dirichlet series to the modified generalized convergence class by its coefficients we need three lemmas.

Lemma 2. If $\alpha \in L^{0}$ and $\beta \in L^{0}$ then for entire Dirichlet series (1) correlation (6) is equivalent to

$$
\int_{\sigma_{0}}^{+\infty} \frac{\alpha\left(\lambda_{\nu(\sigma, F)}\right)}{\beta(\sigma)} d \sigma<+\infty
$$

Indeed, using (8) we have

$$
\ln \mu(\sigma, F)-\ln \mu(0, F)=\int_{0}^{\sigma} \lambda_{\nu(t, F)} d t \leq \sigma \lambda_{\nu(\sigma, F)}
$$

and

$$
\ln \mu(\sigma, F)-\ln \mu(0, F) \geq \int_{\sigma / 2}^{\sigma} \lambda_{\nu(t, F)} d t \geq \frac{1}{2} \sigma \lambda_{\nu(\sigma / 2, F)}
$$

whence the necessary result follows. 
Majorant of Newton for Dirichlet series (see [9, p.180-183] and [3]) also plays a part.

Lemma 3. Let

$$
F^{0}(s)=\sum_{n=0}^{+\infty} a_{n}^{0} e^{s \lambda_{n}}
$$

be the majorant of Newton for entire Dirichlet series (1). Then $\mu\left(\sigma, F^{0}\right)=\mu(\sigma, F), \nu\left(\sigma, F^{0}\right)=$ $\nu(\sigma, F), \varkappa_{n}\left(F^{0}\right) \nearrow+\infty$ as $n \rightarrow+\infty$ and $\left|a_{n}\right| \leq a_{n}^{0}$ for all $n \geq 1$.

Let $p>1, q=\frac{p}{p-1}$ and the function $f$ be positive on $(A, B),-\infty \leq A<B \leq+\infty$. If $\left(\lambda_{n}^{*}\right)$ is a sequence of positive number and $\left(c_{n}\right)$ is a sequence of numbers from $(A, B)$ then

$$
C_{n}:=\frac{\lambda_{1}^{*} c_{1}+\cdots+\lambda_{n}^{*} c_{n}}{\lambda_{1}^{*}+\cdots+\lambda_{n}^{*}} \in(A, B)
$$

It is proved [3] the following generalization of classical Hardy's inequality.

Lemma $4([3])$. Let $p>1$ and $\frac{1}{p}+\frac{1}{q}=1$. If the function $f^{1 / p}$ is convex on $(A, B)$ and the sequence $\left(\mu_{n}\right)$ is positive and nonincreasing then for each $\omega \leq+\infty$

$$
\sum_{n=1}^{\omega} \mu_{n} \lambda_{n}^{*} f\left(C_{n}\right) \leq q^{p} \sum_{n=1}^{\omega} \mu_{n} \lambda_{n}^{*} f\left(c_{n}\right) .
$$

Using Theorem 2 and Lemmas 2-4, we prove the following main theorem.

Theorem 3. Let $\alpha \in L^{0}$ be a concave function on $\left[x_{0},+\infty\right)$ and $\beta(x)=x \gamma(x)$, where $\gamma \in L^{0}$ and

$$
1+\frac{x \gamma^{\prime}(x)}{\gamma(x)} \geq h>0, \quad x \geq x_{0},
$$

Suppose that condition (3) holds and the exponents $\lambda_{n}$ of entire Dirichlet series (1) satisfy condition (7). In order that series (1) belong to modified generalized convergence $\alpha \beta$-class, it is necessary and in the case, when $\varkappa_{n}(F) \nearrow+\infty, n \rightarrow+\infty$, it is sufficient that

$$
\sum_{n=1}^{+\infty}\left(\alpha\left(\lambda_{n}\right)-\alpha\left(\lambda_{n-1}\right)\right) \gamma_{1}\left(\frac{1}{\lambda_{n}} \ln \frac{1}{\left|a_{n}\right|}\right)<+\infty, \quad \gamma_{1}(x)=\int_{x}^{+\infty} \frac{d \ln \sigma}{\gamma(\sigma)} .
$$

Proof. At first, we remark that condition (3) implies

$$
\int_{x_{0}}^{+\infty} \frac{d \ln x}{\gamma(x)} d x=\int_{x_{0}}^{+\infty} \frac{1}{\beta(x)} d x<+\infty
$$

Therefore, in view of Theorem 2 and Lemma 2 it is necessary to find conditions under which (9) holds. Since by Lemma $3 \nu\left(\sigma, F^{0}\right)=\nu(\sigma, F)$ and for the majorant of Newton (10) $\varkappa_{n}\left(F^{0}\right) \nearrow+\infty$ as $n \rightarrow+\infty$, we have

$$
\int_{\sigma_{0}}^{+\infty} \frac{\alpha\left(\lambda_{\nu(\sigma, F)}\right)}{\beta(\sigma)} d \sigma=\int_{\sigma_{0}}^{+\infty} \frac{\alpha\left(\lambda_{\nu\left(\sigma, F^{0}\right)}\right)}{\sigma \gamma(\sigma)} d \sigma=\sum_{n=1}^{+\infty} \int_{\varkappa_{n-1}\left(F^{0}\right)}^{\varkappa_{n}\left(F^{0}\right)} \frac{\alpha\left(\lambda_{\nu\left(\sigma, F^{0}\right)}\right)}{\sigma \gamma(\sigma)} d \sigma+\text { const }=
$$




$$
\begin{gathered}
=\sum_{n=1}^{+\infty} \alpha\left(\lambda_{n}\right) \int_{\varkappa_{n-1}\left(F^{0}\right)}^{\varkappa_{n}\left(F^{0}\right)} \frac{d \ln \sigma}{\gamma(\sigma)}+\text { const }=\sum_{n=1}^{+\infty} \alpha\left(\lambda_{n}\right)\left(\gamma_{1}\left(\varkappa_{n-1}\left(F^{0}\right)\right)-\gamma_{1}\left(\varkappa_{n}\left(F^{0}\right)\right)\right)+\text { const }= \\
=\sum_{n=1}^{+\infty}\left(\alpha\left(\lambda_{n}\right)-\alpha\left(\lambda_{n-1}\right)\right) \gamma_{1}\left(\varkappa_{n-1}\left(F^{0}\right)\right)+\text { const. }
\end{gathered}
$$

From conditions (12) and $\gamma \in L^{0}$ we obtain

$$
\begin{aligned}
& \left(\gamma(x)+x \gamma^{\prime}(x)\right) \gamma_{1}(x)=\left(\gamma(x)+x \gamma^{\prime}(x)\right) \int_{x}^{+\infty} \frac{d \ln \sigma}{\gamma(\sigma)} \geq\left(\gamma(x)+x \gamma^{\prime}(x)\right) \int_{x}^{2 x} \frac{d \ln \sigma}{\gamma(\sigma)} \geq \\
& \geq \frac{\gamma(x)+x \gamma^{\prime}(x)}{\gamma(2 x)} \ln 2 \geq \frac{\gamma(x)+x \gamma^{\prime}(x)}{c \gamma(x)} \ln 2=\frac{\ln 2}{c}\left(1+\frac{x \gamma^{\prime}(x)}{\gamma(x)}\right) \geq \frac{h \ln 2}{c}>0 .
\end{aligned}
$$

We choose $p>1$ such that $\frac{p-1}{p}<\frac{h \ln 2}{c}$. Then

$$
\begin{gathered}
\left(\gamma^{1 / p}(x)\right)^{\prime \prime}=\frac{1}{p} \gamma_{1}^{1 / p-2}(x)\left\{\gamma_{1}(x) \gamma_{1}^{\prime \prime}(x)-\frac{p-1}{p}\left(\gamma_{1}^{\prime}(x)\right)^{2}\right\}= \\
=\frac{1}{p} \gamma_{1}^{1 / p-2}(x)\left\{\gamma_{1}(x) \frac{\gamma(x)+x \gamma^{\prime}(x)}{x^{2} \gamma^{2}(x)}-\frac{p-1}{p} \frac{1}{x^{2} \gamma^{2}(x)}\right\}= \\
=\frac{\gamma_{1}^{1 / p-2}(x)}{x^{2} \gamma^{2}(x)}\left\{\left(\gamma(x)+x \gamma^{\prime}(x)\right) \gamma_{1}(x)-\frac{p-1}{p}\right\} \geq \frac{\gamma_{1}^{1 / p-2}(x)}{x^{2} \gamma^{2}(x)}\left\{\frac{h \ln 2}{c}-\frac{p-1}{p}\right\}>0,
\end{gathered}
$$

i. e. the function $\gamma^{1 / p}(x)$ is convex on $\left[x_{0},+\infty\right)$.

For simplicity, we suppose that in series $(10) a_{0}^{0}=1$. Then for other coefficients we have

$$
\begin{gathered}
\ln a_{n}^{0}=\ln a_{n}^{0}-\ln a_{n-1}^{0}+\cdots+\ln a_{1}^{0}-\ln a_{0}^{0}+\ln a_{0}^{0}= \\
=-\varkappa_{n-1}\left(F^{0}\right)\left(\lambda_{n}-\lambda_{n-1}\right)-\cdots-\varkappa_{0}\left(F^{0}\right)\left(\lambda_{1}-\lambda_{0}\right),
\end{gathered}
$$

i. e.

$$
\frac{1}{\lambda_{n}} \ln \frac{1}{a_{n}^{0}}=\frac{\varkappa_{0}\left(F^{0}\right) \lambda_{1}^{*}+\cdots+\varkappa_{n-1}\left(F^{0}\right) \lambda_{n}^{*}}{\lambda_{1}^{*}+\cdots+\lambda_{n}^{*}}, \quad \lambda_{n}^{*}=\lambda_{n}-\lambda_{n-1} .
$$

Since the function $\alpha$ is concave, the sequence $\mu_{n}=\frac{\alpha\left(\lambda_{n}\right)-\alpha\left(\lambda_{n-1}\right)}{\lambda_{n}-\lambda_{n-1}}$ is nonincreasing. Therefore, by Lemma 4 with

$$
f(x)=\gamma_{1}(x), \lambda_{n}^{*}=\lambda_{n}-\lambda_{n-1}, C_{n}=\frac{1}{\lambda_{n}} \ln \frac{1}{a_{n}^{0}}
$$

and $c_{n}=\varkappa_{n-1}\left(F^{0}\right)$, we have

$$
\begin{gathered}
\sum_{n=1}^{+\infty}\left(\alpha\left(\lambda_{n}\right)-\alpha\left(\lambda_{n-1}\right)\right) \gamma_{1}\left(\frac{1}{\lambda_{n}} \ln \frac{1}{a_{n}^{0}}\right)=\sum_{n=1}^{+\infty} \mu_{n} \lambda_{n}^{*} f\left(C_{n}\right) \leq \\
\leq\left(\frac{p}{p-1}\right)^{p} \sum_{n=1}^{+\infty} \mu_{n} \lambda_{n}^{*} f\left(c_{n}\right)=\left(\frac{p}{p-1}\right)^{p} \sum_{n=1}^{+\infty}\left(\alpha\left(\lambda_{n}\right)-\alpha\left(\lambda_{n-1}\right)\right) \gamma_{1}\left(\varkappa_{n-1}\left(F^{0}\right)\right) .
\end{gathered}
$$

If (9) holds then (14) and (16) imply (13) with $a_{n}^{0}$ instead of $\left|a_{n}\right|$. 
On the contrary, (15) implies $\frac{1}{\lambda_{n}} \ln \frac{1}{a_{n}^{0}} \leq \varkappa_{n-1}\left(F^{0}\right)$. And if (13) holds with $a_{n}^{0}$ instead $\left|a_{n}\right|$ then in view of (14) we obtain (9) because the function $\gamma_{1}$ is decreasing.

Theorem 3 is proved for the majorant of Newton. Therefore, condition (13) with $a_{n}^{0}$ instead of $\left|a_{n}\right|$ is necessary and sufficient for the belonging of $F^{0}$ to the modified generalized convergence $\alpha \beta$-class.

Since $\left|a_{n}\right| \leq a_{n}^{0}$, we obtain the necessity of condition (13). If the sequence $\left(\varkappa_{n}(F)\right)$ does not decrease then $\left|a_{n}\right|=a_{n}^{0}, \varkappa_{n}\left(F^{0}\right)=\varkappa_{n}(F)$ and condition (13) is sufficient. The proof of Theorem 3 is complete.

Remark 1. Since $\beta(x)=x \gamma(x)$, the conditions $\alpha \in L^{0}$ and $\ln n=O\left(\lambda_{n} \gamma^{-1}\left(\alpha\left(\lambda_{n}\right)\right)\right)$ as $n \rightarrow+\infty$ in Theorem 3 are much weaker than the condition $\alpha\left(e^{x}\right) \in L^{0}$ and $\ln n=$ $o\left(\lambda_{n} \beta^{-1}\left(\alpha\left(\lambda_{n}\right)\right)\right)$ as $n \rightarrow+\infty$ in Theorem 1 .

Remark 2. In a general case, the following condition

$$
\ln n=O\left(\lambda_{n} \gamma^{-1}\left(\alpha\left(\lambda_{n}\right)\right)\right)
$$

as $n \rightarrow+\infty$ in Theorem 3 cannot be relaxed. Indeed, let for example $\alpha(x) \equiv x$ and $\gamma(x) \equiv$ $x^{p-1}, p>1$, for $x \geq x_{0}$. Then $\lambda_{n} \gamma^{-1}\left(\alpha\left(\lambda_{n}\right)\right)=\lambda_{n}^{p /(p-1)}$ and condition (5) has the form

$$
\int_{\sigma_{0}}^{+\infty} \frac{\ln M(\sigma, F)}{\sigma^{p+1}} d \sigma<+\infty .
$$

It is proved [10] that the last condition is equivalent to the condition

$$
\int_{\sigma_{0}}^{+\infty} \frac{\ln \mu(\sigma, F)}{\sigma^{p+1}} d \sigma<+\infty
$$

for every Dirichlet series with given sequence of exponents $\left(\lambda_{n}\right)$ if and only if

$$
\ln n=O\left(\lambda_{n}^{p /(p-1)}\right) \quad(n \rightarrow+\infty) .
$$

\section{REFERENCES}

1. G. Valiron, General theory of integral functions, Toulouse, 1923, $382 \mathrm{p}$.

2. P.K. Kamthan, A theorem of step functions. II, Instambul univ. fen. fac. mecm. A. 28 (1963), 65-69.

3. O.M. Mulyava, On convergence classes of Dirichlet series, Ukr. Math. J., 51 (1999), №1, 1485-1494. (in Ukrainian)

4. O.M. Mulyava, Convergence classes in theory of Dirichlet series, Dopov. Nac. acad. Ukr., (1999), №3, 35-39. (in Ukrainian)

5. M.N. Sheremeta, Connection between the growth of the maximum of the modulus of an entire function and the moduli of the coefficients of its power series expansion, Izv. Vyssh. Uchebn. Zaved. Mat., (1967), №2, 100-108. (in Russian)

6. M.M. Sheremeta, Entire Dirichlet series, K.: ISDO, (1993), 168 p. (in Ukrainian)

7. M.M. Sheremeta, On two classes of positive functions and the belonging of main characteristics of entire functions then, Mat. Stud., 19 (2003), №1, 75-82. 
8. E. Seneta, Regularly varying functions, Lecture Notes in Mathematics, V.508, Springer-Verlag, Berlin, 1976, 112 p.

9. A.F. Leont'ev, Series of exponents, M.: Nauka, 1956, 536 p. (in Russian)

10. O.M. Mulyava, M.M. Sheremeta, On the belonging of entire Dirichlet series to logarithmic convergence class, Mat. Stud., 3 (2010), №1, 17-21. (in Ukrainian)

Kyiv National University of Food Technologies info@nuft.edu.ua

Received 5.01.2018

Revised 26.10.2018 